\title{
Caracterización de la producción científica sobre clasificaciones de universidades. Un estudio bibliométrico desde 1988 a 2018
}

\author{
Andrea King-Domínguez ${ }^{(1)}$, Xavier Llinàs-Audet( ${ }^{(2)}$ y Luis Améstica-Rivas( ${ }^{(3)}$ \\ (1) Departamento Economía y Finanzas, Facultad de Ciencias Empresariales, Universidad del Bío-Bío, Casilla 447, \\ Chillán, Chile (correo-e: aking@ubiobio.cl) \\ (2) Universidad Politécnica de Cataluña, Facultad de Informática de Barcelona, Cátedra UNESCO de Dirección \\ Universitaria, Campus Nord - Edif. C5, C. Jordi Girona, 1- 3, 08034 Barcelona, España. \\ (correo-e: xavier.llinas@upc.edu) \\ (3) Departamento Gestión Empresarial, Facultad de Ciencias Empresariales, Universidad del Bío-Bío, Casilla 447, \\ Chillán, Chile (correo-e: lamestica@ubiobio.cl)
}

Recibido Jul. 12, 2019; Aceptado Ago. 28, 2019; Versión final Oct. 18, 2019, Publicado Abr. 2020

\begin{abstract}
Resumen
El objetivo de este trabajo es describir la evolución de la investigación sobre los sistemas de clasificación de universidades (university rankings). Las clasificaciones de universidades permiten comparar e informar acerca de la calidad de la actividad académica de las instituciones de educación superior. Luego de la publicación de la primera clasificación global el año 2004, ranking de Shanghái, se han ido desarrollando diversas clasificaciones universitarias e incentivando una mayor discusión científica sobre el tema. Para el estudio se utilizó como base de datos los artículos de esta temática publicados en la Web of Science (WoS). Se aplicaron diversos indicadores bibliométricos que permitieron detectar las principales revistas, organizaciones y autores que han trabajado en el tema. A partir de un estudio de co-palabras se evidenciaron las principales líneas de investigación, destacándose entre ellas la medición de la productividad de la investigación.
\end{abstract}

Palabras clave: clasificación de universidades; bibliometría; análisis de citas; análisis de co-palabras

\section{Characterization of the scientific production on university ranking. A bibliometric study from 1988 to 2018}

\begin{abstract}
The aim of this paper is to describe the evolution of research and publications about university rankings. University rankings allows comparing the quality of the academic activity of higher education institutions. After the publication of the first global ranking in 2004, the Shanghai ranking, various university classifications have been developed and a greater scientific discussion on the subject has been encouraged. For this study, the articles on the subject published on the Web of Science (WoS) were used as a database. Several bibliometric indicators were applied to detect the main journals, organizations and authors that have worked on the subject. From a study of co-words, the main lines of research were determined, highlighting among them the measurement of research productivity.
\end{abstract}




\section{INTRODUCCIÓN}

De acuerdo a lo señalado por Barra (2019), los retos de la educación superior (ES) del siglo XXI se fundamentan en principios de excelencia, calidad y pertinencia, siendo la excelencia académica uno de los elementos de mayor importancia en la ES. Es en este contexto que uno de los instrumentos para monitorear ésta se encuentra en los llamados rankings, de una amplia difusión y heterogeneidad. Los rankings universitarios comparan y ordenan a las instituciones de ES de manera descendente, según un conjunto de criterios e indicadores de calidad. De ahí que los rankings han sido considerados como instrumentos de transparencia y que entregan información relevante, fiable y válida a las partes interesadas, como son los estudiantes y sus padres, académicos, administradores, organizaciones privadas y púbicas y al gobierno, entre otros (King-Domínguez et al., 2018). Es más, tal como señala O'Loughlin et al. (2015), estas clasificaciones han servido de base para la financiación y las decisiones políticas de los gobiernos respecto a sus universidades y han permitido evaluar la calidad académica general de las instituciones y su reputación, especialmente en lo relativo a la investigación científica.

En cuanto a su origen, Rauhvargers (2011) y Salmi y Saroyan (2007), señalan que las primeras clasificaciones de instituciones de ES fueron realizadas entre los años 1870 y 1890 por la Commission of the U.S. Bureau of Education. Posteriormente se generaron distintos sistemas de clasificación universitaria en varios países del mundo. Como ejemplo de ello se puede mencionar el ranking "American Best Colleges" publicado en Estados Unidos por primera vez en 1983 por The U.S. News and World Report. Igualmente, en Alemania desde 1998 el Centro para el Desarrollo de la Educación (Centrum für Hochschulentwicklung- CHE) ha ofrecido un sistema de indicadores de calidad de las universidades. En otras latitudes, destaca el trabajo realizado desde el año 2002 en Pakistán, donde se ha desarrollado un ranking universitario cuyo fin es promover el desarrollo rápido e integral de todo el sistema de educación terciaria.

Sin embargo, fue en el año 2003 que se publicó el primer ranking global universitario: el Academic Ranking of World Universities (ARWU o Ranking de Shanghái). Después de esta iniciativa otras entidades han elaborado rankings universitarios globales basados en datos académicos internacionalmente comparables (Buela-Casal et al., 2007). Cada uno utiliza distintas metodologías, criterios de evaluación e indicadores, haciendo que exista una gran variedad de tipos de clasificaciones. Rauhvargers (2011) señala que los rankings globales pueden agruparse en cuatro categorías según su objeto de medición: aquellos orientados a proporcionar tablas de universidades de clase mundial; los rankings que se enfocan en los resultados de investigación; los que miden el impacto en la web y los que hacen comparaciones entre universidades. En el primer grupo estarían los rankings ARWU, QS World University Ranking (QS), Times Higher Education World University Rankings (THE), Best Global University Rankings - U.S. News and World Report (BGUR). EI segundo grupo estaría integrado por NTU Ranking (NTU); University Ranking by Academic Performance (URAP); Scimago Institutions Rankings (SIR); CWTS Lieden Ranking y Nature Index. Un ejemplo de los rankings del tercer y cuarto grupo serían Webometrics y U-Multirank, respectivamente.

Es también a partir de la última década del siglo XX que comienzan a surgir investigaciones de carácter científico en torno a los rankings. Hasta el año 2004 las publicaciones sobre RU eran esporádicas, aumentando de manera significativa en los años posteriores y hasta el presente. Es así que entre 1988 y 2018 se han publicado en revistas indexadas en la WoS 557 artículos directamente relacionados con este tema. En atención a lo anterior, y considerando la importancia que ha tomado el tema de los RU en los últimos años, es que se ha planteado como objetivo de estudio conocer y describir la evolución que han tenido las investigaciones en esta área, a través de un análisis bibliométrico.

\section{METODOLOGÍA}

Para el análisis del estado general de la investigación sobre RU se utilizaron técnicas bibliométricas. Para ello se recurrió a la base de datos de la WoS, utilizándose la alternativa "colección principal de la Web of Science". Con respecto al criterio de búsqueda, se utilizó la alternativa "tema", la que realiza exploraciones en el título, resumen y palabras clave, seleccionándose únicamente artículos. En las exploraciones realizadas no se especificó el período de estudio, entregando el sistema todos los artículos publicados entre los años 1988 y 2018. Se realizaron distintas exploraciones, las que incluyeron la búsqueda de los siguientes términos en orden de prelación. Los conceptos de la primera exploración fueron: "university ranking*" or "university league table*" or "ranking of university" or "rankings of universities*"; Luego, con el fin de tener certeza de que se han incorporado en la base de datos todos los artículos referidos a los rankings globales, si hicieron exploraciones específicas a cada ranking global mencionado en la introducción. Así la siguiente exploración fue: "Shanghai ranking*" or "Shanghai league table*" or "Shanghai university rank".Las siguientes exploraciones fueron similares a la segunda, pero para distintos rankings y sus acrónimos (ARWU; CWTS; BGUR; NTU; QS; THE, SIR; U-Multirank; URAP y Webometrics). Como resultado de esta etapa, el sistema arrojó un total de 842 publicaciones, que incorporaban a 676 artículos. Cada artículo fue revisado con el fin 
de verificar la presencia de las palabras clave utilizadas en las distintas búsquedas. Finalmente, se obtuvo una base de datos que incluía a 557 artículos publicados entre los años 1988 y 2018. En una segunda etapa, los datos entregados por la WoS se vaciaron tanto a un archivo Excel como a uno de texto, lo que permitió aplicar el software de libre disposición VOSviewer. Este software permite crear mapas basados en datos de red, lo cual facilita su visualización y exploración (van Eck y Waltman, 2017).

A partir de la información recolectada, y sobre la base de la metodología aplicada en los estudios de Herrera Madueño et al. (2015) y Ortiz-de Urbina-Criado et al. (2018), se realizó un estudio descriptivo con el fin de analizar la evolución que ha tenido a lo largo de los años la investigación sobre esta temática. Para ello fue necesario definir el número de artículos, autores, revistas y organizaciones que han publicado sobre RU en cada año, así como el número de referencias utilizadas. A partir de la información recopilada, se calcularon también indicadores bibliométricos como el número de autores por artículo, número de artículos por revistas y de autores por organización. La siguiente etapa consistió en analizar detalladamente la información recogida.

Primero, en relación a las revistas, se determinaron aquellas que pueden considerarse centrales en el campo de la investigación en RU. Para ello, siguiendo la metodología aplicada por Huang y Ho (2011), se aplicó la ley de Bradford que postula que si se ordenan las revistas por orden decreciente de productividad de artículos sobre un determinado tema, ellas pueden dividirse en grupos o zonas, cada uno con aproximadamente el mismo número de artículos. En la primera zona o núcleo, estaría un pequeño número de revistas que se consideran medulares en el campo estudiado, ya que concentran un gran número de artículos. En contraste, la última zona agruparía un gran número de revista con pocas publicaciones cada una (Tsay y Li, 2017). Luego se investigó cuáles son las áreas de interés de las revistas en su conjunto, y en especial de aquellas pertenecientes a la zona núcleo, según la clasificación de la WoS. Segundo, se expusieron las principales organizaciones que investigan sobre $\mathrm{RU}$, en cuanto a número de artículos publicados en la WoS. Adicionalmente se determinó el país al cual pertenecen y la colaboración que existe entre ellas. Esto último se realizó por medio de la aplicación del software Vosviewer, considerando que las organizaciones debían tener al menos cinco publicaciones. Tercero, se determinaron los autores más relevantes en el tema, tanto por el número de artículos publicados como por el número de citas recibidas. Igualmente se definió cuáles son las redes de trabajo entre autores, considerando únicamente aquellos autores que han publicado como mínimo cuatro artículos. Cuarto, para conocer los fundamentos sobre los cuales se ha investigado sobre RU, se estudiaron las referencias más utilizadas por los estudios seleccionados.

Finalmente, se hizo un estudio descriptivo de las palabras clave utilizadas en los distintos artículos, junto a un análisis de co-palabras. El objetivo de este estudio es revelar los patrones y tendencias del discurso científico, y medir la fuerza de asociación de términos representativos de las publicaciones. Según Huang y Ho (2011), esto facilita el análisis de información acerca de los temas que preocupan a la mayoría de los investigadores. El análisis incorporó tanto las palabras clave aplicadas por los autores como las sugeridas por las WoS. Previo al estudio, siguiendo la metodología aplicada por Ortiz-de Urbina-Criado et al. (2018), se realizó un proceso de estandarización de conceptos, relacionado al uso de abreviaturas, utilización de ortografía alternativa y fusión de términos sinónimos. El análisis de palabras clave y co-palabras se realizó con el software VOSviewer, solicitando que el mínimo de ocurrencia de las palabras clave fuera de 10. Con el fin de facilitar la interpretación de los resultados, cada clúster se subdividió en conglomerados.

\section{RESULTADOS Y DISCUSIÓN}

De acuerdo a la información entregada por la WoS, y como se indica en la Tabla 1, existen publicaciones sobre RU desde el año 1988.

Tabla 1. Caracterización de las publicaciones en RU, período 1988-2018

\begin{tabular}{|l|c|c|c|}
\hline & $1988-2003$ & $2004-2018$ & Total \\
\hline$N^{\circ}$ de artículos & 13 & 544 & 557 \\
\hline $\mathrm{N}^{\circ}$ de autores & 23 & 1.024 & 1049 \\
\hline $\mathrm{N}^{\circ}$ de revistas & 13 & 281 & 291 \\
\hline $\mathrm{N}^{\circ}$ de organizaciones & 13 & 594 & 598 \\
\hline $\mathrm{N}^{\circ}$ de referencias & 222 & 15.148 & 15.348 \\
\hline $\mathrm{N}^{\circ}$ autores por artículo & 2,1 & 1,9 & 1,9 \\
\hline $\mathrm{N}^{\circ}$ de artículos por revista & 1,0 & 1,9 & 1,9 \\
\hline $\mathrm{N}^{\circ}$ de autores por organización & 2,2 & 1,7 & 1,8 \\
\hline
\end{tabular}




\section{Evolución sobre la base de los artículos publicados por año y revistas}

A partir del año 1988 hasta el 2003, la producción científica en esta temática era esporádica, registrándose 13 artículos. A partir del 2004 comienza un período de mayor productividad. Es así que en el período 20042018 se publicaron 544 artículos, lo que equivale al $97,7 \%$ del total. El aumento del número de artículos por año ha ido de la mano del crecimiento de otros indicadores, como el número de autores, revistas y organizaciones que trabajan sobre el tema. En relación a las revistas, 291 han publicado artículos relacionados a los RU. Se observa que durante el primer período (1988-2003) hubo el mismo número de revistas que de artículos, entregando una relación de artículos por revistas igual a 1,0. En los años siguientes esta relación aumenta a 1,9. Lo anterior indica que un número importante de ellas han publicado más de un artículos sobre la temática.

\section{Principales revistas}

Luego de ordenarse las revistas de manera decreciente según el número de artículos que han publicado, siguiendo la metodología de la ley de Bradford (Huang y Ho, 2011), se formaron tres grupos, cada uno con un número similar de artículos. Se verificó que 12 revistas contienen aproximadamente un tercio del total de publicaciones (185) y, por lo tanto, conformarían la zona núcleo. Por otra parte, los dos tercios restantes se encuentran difundidos en 279 revistas. De esta forma se pudo constatar cuáles son las revistas más relevantes en cuanto a número de artículos publicados en $\mathrm{RU}$.

En lo referente a las revistas de la zona núcleo, sobresalen por el alto número de artículos publicados las revistas Scientometrics con 78 artículos (14,0\%), y Higher Education con 24 artículos (4,3\%). Adicionalmente, se puede señalar que, a excepción de la revista Current Science (de origen Indio), todas las revistas del núcleo son europeas. En este grupo destacan las revistas españolas Revista Española de Documentación Científica, junto a Profesional de la Información. De los Países Bajos destacan Higher Education, Journal of Cleaner Production, Journal of Informetrics y Scientometrics; del Reino Unido son las revistas Higher Education Policy; Journal of the Association for Information Science and Technology; Research Evaluation y Studies in Higher Education; y de Rusia es la revista Sotsiologicheskie Issledovanya (table 2).

Tabla 2. Principales revistas según número de artículos publicados en RU: período 1988- 2018

\begin{tabular}{|c|c|c|c|}
\hline Revista & $\begin{array}{l}\text { Artículos } \\
\text { publicados } \\
\text { sobre } R U\end{array}$ & Categorías WoS y Cuartil según categoría WoS (2018) & $\begin{array}{l}\text { Factor de } \\
\text { Impacto } \\
\text { (2018) }\end{array}$ \\
\hline Scientometrics & 78 & $\begin{array}{l}\text { - Ciencias de la computación, aplicaciones interdisciplinarias (Q2) } \\
\text { - Ciencias de la información y ciencias de la biblioteca (Q1) }\end{array}$ & 2,770 \\
\hline Higher Education & 24 & - Educación e investigación educativa (Q1) & 3,005 \\
\hline $\begin{array}{l}\text { Journal of the } \\
\text { Association for } \\
\text { Information Science } \\
\text { and Technology }\end{array}$ & 15 & - Ciencias de la computación, sistemas de información (Q2) & 3,103 \\
\hline $\begin{array}{l}\text { Studies in Higher } \\
\text { Education }\end{array}$ & 13 & - Educación e investigación educativa (Q1) & 2,854 \\
\hline Journal of Informetrics & 13 & - Ciencias de la computación, aplicaciones interdisciplinarias (Q1) & 3,879 \\
\hline $\begin{array}{l}\text { Revista Española de } \\
\text { Documentación } \\
\text { Científica }\end{array}$ & 8 & - Ciencias de la información y ciencias de la biblioteca (Q3) & 0,985 \\
\hline $\begin{array}{l}\text { Profesional de la } \\
\text { Información }\end{array}$ & 8 & - Ciencias de la información y ciencias de la biblioteca (Q2) & 1,318 \\
\hline $\begin{array}{l}\text { Higher Education } \\
\text { Policy }\end{array}$ & 6 & - Educación e investigación educativa (Q3) & 1,333 \\
\hline Research Evaluation & 5 & - Ciencias de la información y ciencias de la biblioteca (Q1) & 2,875 \\
\hline $\begin{array}{l}\text { Journal of cleaner } \\
\text { Production }\end{array}$ & 5 & $\begin{array}{l}\text { - Ciencia y tecnología verde y sostenible (Q1) } \\
\text { - Ingeniería ambiental (Q1) } \\
\text { - Ciencias ambientales (Q1) }\end{array}$ & 6,395 \\
\hline Current Science & 5 & - Ciencias multidisciplinarias (Q3) & 0,756 \\
\hline $\begin{array}{l}\text { Sotsiologicheskie } \\
\text { Issledovaniya }\end{array}$ & 5 & - Sociología (Q4) & 0,619 \\
\hline
\end{tabular}


Igualmente, en las revistas de la zona núcleo se encuentran publicados artículos de alto impacto. Como ejemplo se pueden mencionar la revista Scientometrics, que publicó los siguientes artículos: Aguilló et al. (2010); Billaut et al. (2010); Buela-Casal et al. (2007) y Van Raan (2005), cada uno con 107, 83, 94 y 367 citas. En las revistas Higher Education y Journal of Association for Informatics Science and Technology, que cuentan con 24 y 15 artículos cada uno, están publicados los artículos Dill y Soo (2005) y Thelwall y Kousha (2008), cada uno con 237 y 84 citas.

Según la clasificación dada por la WoS, los ámbitos sobre los que publican las revistas que pertenecen al núcleo son diversos, aunque predominan aquellos relacionados a las ciencias de la computación y de la informática. Al analizarse las 287 revistas seleccionadas, el mayor número publica en la categoría de "educación e investigación educativa", seguida por "ciencias de la información", y "ciencias de la biblioteca y ciencias de la computación". Otras áreas de interés son "economía y negocios", "sociología", y "ciencias sociales y otros temas", entre otras.

\section{Productividad por organizaciones}

En el total de publicaciones analizadas han participado 598 organizaciones, destacando entre ellas la Universidad de Granada con 20 artículos, seguida por la Universidad de Belgrado y la Sociedad Max Planck para la Promoción de la Ciencia (Max-Planck-Gesellschaft zur Förderung der Wissenschaften), cada una con 13 artículos, la Universidad Carlos III de España, con 12 artículos, y las universidades de Leiden y de Vigo con 11 artículos cada una.

Junto al análisis de las organizaciones que mayor aporte han realizado a la investigación sobre RU, es interesante detectar cuál es la colaboración entre ellas. Por medio de la aplicación del software VOSviewer se obtuvieron cuatro grupos de organizaciones que al menos habían publicado cinco artículos. En el primer grupo están las universidades de Belgrado y de Ámsterdam, junto a la Sociedad Max Planck para la Promoción de la Ciencia, el Consejo Superior de Investigaciones Científicas (CSIC) y la Escuela Politécnica Federal de Zúrich (Eidgenössische Technische Hochschule Zürich-ETH). El segundo grupo se conforma por la Universidad de Vigo y Universidad Nacional Australiana. En el tercer grupo están las universidades de Leiden y Carlos III y en el cuarto las universidades de Granada y Navarra.

\section{Productividad de los países e idioma de publicación}

En términos geográficos, las publicaciones sobre RU se concentran principalmente en Europa (340 artículos, 61\%), seguido por Asia (107 artículos, 19,2\%), América (94 artículos, 16,9\%), Oceanía (29 artículos, 5,2\%) y África (16 artículos, 2,9\%). En cuanto a los países, en Europa se desatacan España con 79 artículos (14,2\%) y Reino Unido con 56 artículos (10,1\%). En Asia se destacan China y Taiwán, con 34 y 16 documentos cada uno $(6,1 \%$ y $2,9 \%$ respectivamente). En los continente de América, Oceanía y África, la producción científica en la temática se concentra en un país: Estados Unidos (61 artículos), Australia (27 artículos) y Sudáfrica (11 artículos), respectivamente. En cuanto al idioma de las publicaciones, a pesar de que en los países que han investigado sobre RU se habla una diversidad de idiomas, en 491 documentos se ha utilizado el inglés $(88,2 \%)$, seguido por el español (39 documentos, $7,0 \%$ ) y ruso (20 documentos, $3,6 \%$ ).

\section{Principales autores y co-autorías}

En total, 1.049 investigadores han participado en estudios sobre rankings universitarios, 23 en el primer período y 1.024 en el segundo. De acuerdo al análisis realizado, en el primer período sólo un autor publicó más de un artículo. En contraste, en el segundo período 116 autores han participado en la publicación de más de un artículo, pero sólo tres lo han hecho en 10 o más ocasiones.

El investigador con mayor número de publicaciones en la WoS corresponde al investigador de la Sociedad Max Planck, Dr. Bornmann, quien registra 13 artículos en la temática de RU. En total, estos artículos han sido citados en 199 ocasiones. Por su parte, el Dr. Domingo Docampo, de la Universidad de Vigo, registra 10 artículos, que en conjunto han sido citas 157 veces. Por último, el Dr. Veljok Jeremic de la Universidad de Belgrado registra 10 trabajos en la WoS sobre RU que han sido citados en 169 ocasiones. Entre los autores más citados se encuentra el Dr. Van Raan. Sólo en su trabajo Fatal Attraction: Conceptual and Methodological Problems in the Ranking of Universities by Bibliometric Methods ha recibido 363 citas. También se pueden destacar los investigadores David D. Dill y Maarja Soo. En relación a la co-autoría de los 557 artículos, 397 fueron escritos por más de un autor, lo que equivale al 71,3\%. En cuanto a las redes de co-autoría, considerando sólo aquellos autores que han publicado al menos 4 artículos, es posible distinguir a 2 grupos, que están compuestos por: Grupo 1: Bornmann, I.; Mutz, R.; Daniel, H. D.; Leydesdorff, I.; y Grupo 2: Jeremic, V.; Bulajic, M.; Dobrota, M. y Martic, M. La Tabla 3 indica los principales autores según el número de documentos publicados en el tema y según el número de citas recibidas. Se agrega el índice H de los autores, según el total de publicaciones. 
Tabla 3. Principales autores sobre RU

\begin{tabular}{|l|c|c|c|l|}
\hline Autor & No Doc. & Citas / doc. & $\begin{array}{c}\text { Índice } \\
H\end{array}$ & \multicolumn{1}{|c|}{ Institución } \\
\hline \multicolumn{5}{|c|}{ Principales autores según número de publicaciones } \\
\hline Bornmann, L. & 13 & 15,3 & 41 & Administrative Headquarters of the Max Planck Society \\
\hline Docampo, D. & 10 & 15,7 & 10 & Universidad de Vigo \\
\hline Jeremic, V. & 10 & 16,4 & 12 & University of Belgrade \\
\hline Mutz, R. & 7 & 13,4 & 21 & ETH Zurich \\
\hline Abramo, G. & 6 & 19,0 & - & Istituo di análisis dei sistema ed informática "Antonio Ruberti" \\
\hline Torres-Salinas, D. & 6 & 15,3 & 16 & Universidad de Granada y Universidad de Navarra \\
\hline \multicolumn{5}{|c|}{ Principales autores según número de citas } \\
\hline Dill, D. D. & 1 & 237 & 12 & University of North Carolina at Chapel Hill \\
\hline Van Raan, A.F.J. & 3 & 190,7 & 43 & Universidad de Leiden \\
\hline Burrows, R & 1 & 161 & 15 & Universidad de Londres \\
\hline Piller, I & 1 & 103 & 4 & Universidad Macquarie \\
\hline Saisana, I & 1 & 102 & 13 & European Commission Joint Research Centre \\
\hline Thelwall, M & 1 & 84 & 50 & Wolverhampton University \\
\hline
\end{tabular}

\section{Análisis de Co-citas}

Al analizarse la base de datos relacionada a los 557 artículos de RU, se pudo comprobar que habían sido utilizadas más de 15.000 referencias. De ellas 1.629 han sido citadas por más de un artículo. A partir de este grupo se seleccionaron las 15 más utilizadas, las que se muestran en la Tabla 4. El artículo que más ha sido referenciado es el Van Raan (2005). Aquí el autor analiza los sistemas de clasificación de las instituciones de investigación, y asevera que los rankings son una herramienta inadecuada como herramientas de evaluación de desempeño de la investigación. Agrega que los rankings no son el problema, sino que los indicadores utilizados para la clasificación a menudo no son lo suficientemente avanzados. A su vez, analiza las condiciones que son necesarios para una aplicación exitosa de indicadores bibliométricos avanzados como herramienta de apoyo para la revisión por pares.

Tabla 4. Principales referencias utilizadas en los artículos de RU

\begin{tabular}{|l|c|}
\hline Documento & $\begin{array}{c}N^{\circ} \text { de citas en } \\
\text { artículos de } R U \\
\text { publicados en WoS }\end{array}$ \\
\hline Van Raan (2005) & 85 \\
\hline Dill y Soo (2005) & 63 \\
\hline Liu y Cheng (2005) & 62 \\
\hline Hazelkorn (2011) & 58 \\
\hline Marginson y Wende (2007) & 51 \\
\hline Aguilló et al. (2010) & 52 \\
\hline Waltman et al. (2012) & 46 \\
\hline Saisana et al. (2011) & 42 \\
\hline Salmi (2009) & 42 \\
\hline Hazelkorn (2008) & 36 \\
\hline Billaut et al.(2010) & 35 \\
\hline Hirsch (2005) & 35 \\
\hline Buela-Casal et al. (2007) & 34 \\
\hline Rauhvargers (2011) & 30 \\
\hline Espeland y Suader (2007) & 29 \\
\hline
\end{tabular}

En segundo lugar estaría el artículo Dill y Soo (2005) que ha sido aplicado como referencia en 63 ocasiones por los artículos de RU estudiados. En él los autores se plantean tres preguntas: 1) si existe un consenso internacional emergente sobre la medición de la calidad académica, de la forma en que se refleja en estos sistemas de clasificación; 2) si los diferentes sistemas de clasificación impactan el comportamiento 
universitario y académico en sus respectivos países; y 3) si hay intereses públicos que no se reflejen en estas clasificaciones. Por medio de un análisis comparativo de los rankings universitarios nacionales de Australia, Canadá, Reino Unido y Estados unidos concluyen que las definiciones de calidad académica están convergiendo. Agregan que las políticas de gobierno son contribuyentes aparentemente importantes para las clasificaciones universitarias más efectivas, ya que pueden ayudar a mejorar la calidad de la información disponible para estudiantes y universidades. En contraste, las políticas más liberales aplicadas en Estados Unidos sobre la disponibilidad de información relacionada al rendimiento universitario pueden contribuir a tener sistemas de clasificación de menor validez.

Por último, otro artículo con alto número de citas es el de Liu y Cheng (2005). Este trabajo explica detalladamente la metodología aplicada por el Academic Ranking of World (ARWU) desarrollado por la Shanghai Jiao Tong University. Igualmente, analiza los resultados y alcance del ranking ARWU. Entre los puntos señalados destaca la dificultad para evaluar la calidad de las universidades, por lo que ARWU decidió clasificar las instituciones sobre la base de los resultados en investigación.

\section{Palabras clave y co-palabras}

El resultado del estudio muestra que se han utilizado 1.925 palabras clave, de las cuales 1.436 han sido propuesta por los autores y 653 por la WoS. Del conjunto, se analizaron las 46 que han sido aplicadas en al menos 10 ocasiones. El concepto con mayor número de menciones es "ranking universitario" (university ranking) -el cual estuvo presente en las búsquedas iniciales- con 322 citas, seguido por "educación superior" (higher education) (129), "universidades" (universities) (99) y "rendimiento" (performance) (70). Dentro del conjunto de palabras con mayor número de citas también se encuentran los nombres de países como España (13) y Estados Unidos (12). También han sido aplicados otros nombres de países, continentes o zonas geográficas, siendo mayormente citados aquellos países del continente asiático (47), seguido por los europeos (34) (Tabla 5).

Tabla 5. Clúster y frecuencia de uso de las palabras clave

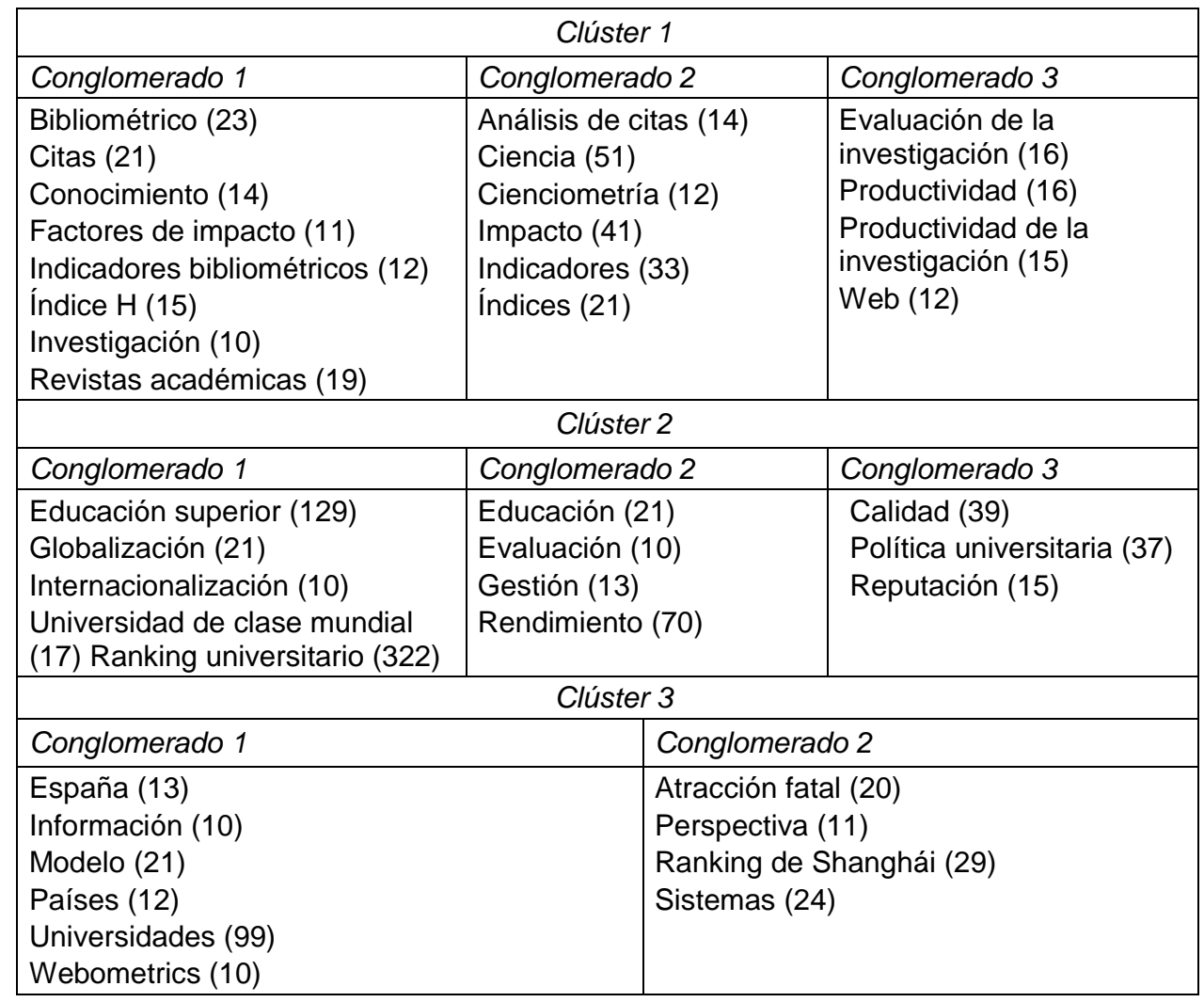

Adicionalmente, el estudio de co-palabras indica que los términos pueden agruparse en tres clúster o conglomerados. Luego, con el fin de facilitar la interpretación y sobre la base de los resultados obtenidos previamente, se hizo una reagrupación de los términos de cada clúster. Los conceptos de cada clúster se muestran en la Tabla 5. Igualmente en la Figura 1 están representados los distintos clúster, identificados cada uno por la vía de diferentes colores. Cada palabra está acompañada de un círculo cuyo tamaño es proporcional al número de registros. Las líneas que unen los círculos representan los enlaces de los registros y la distancia entre las palabras clave indica la proximidad que hay entre pares de ellas. 


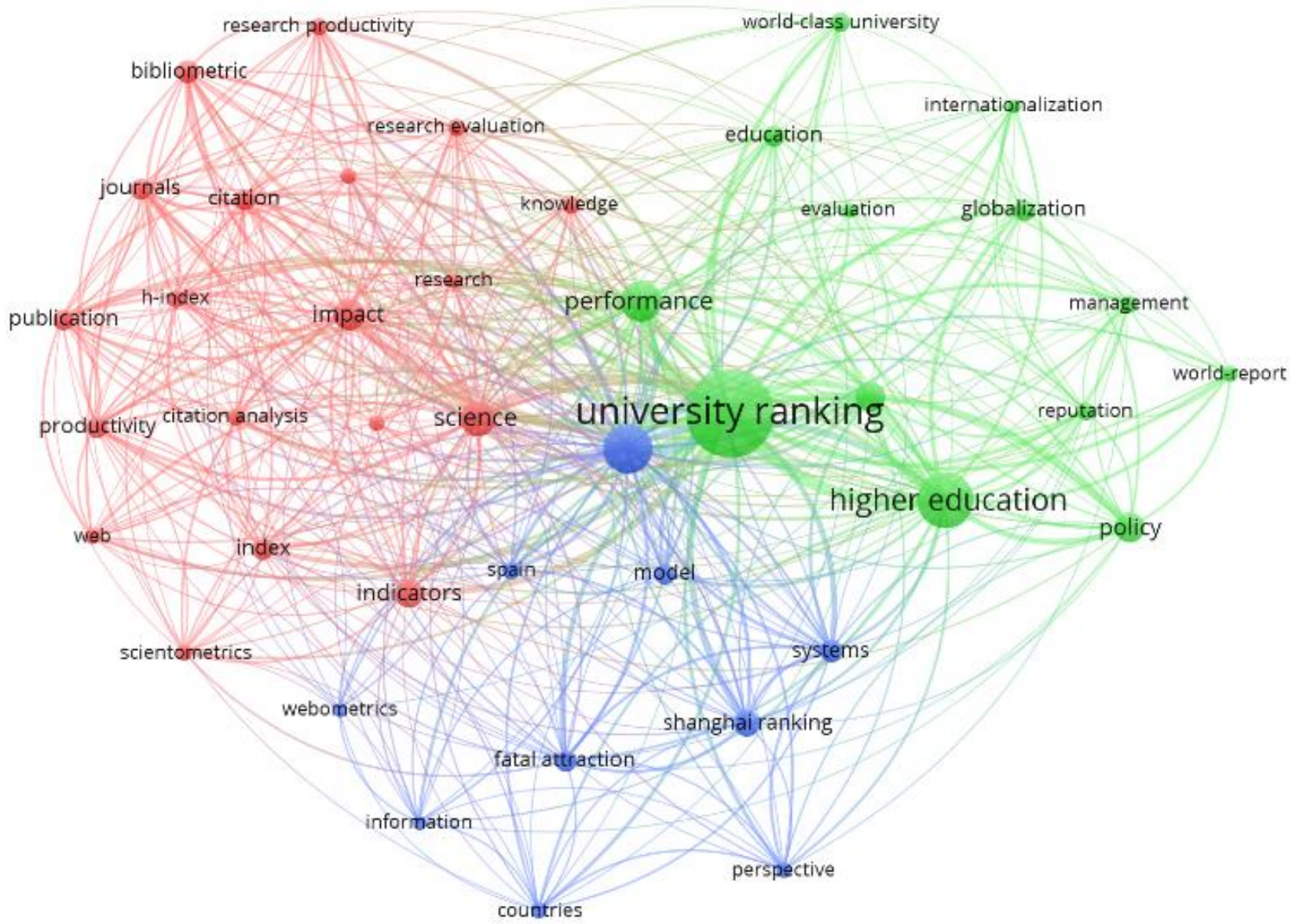

Fig.1: Redes de co-palabras

El primer clúster, de color rojo, incorpora 17 palabras clave, y se subdivide en tres conglomerados. El primero, se relaciona a la medición de la productividad de la investigación (ejemplo, Abramo y D’Angelo, 2014), el segundo al impacto de la investigación (ejemplo: Van Raan, 2005) y el tercero a la divulgación del conocimiento (ejemplo: Torres-Salinas et al. 2011). El segundo clúster, de color verde, tiene 12 palabras clave, agrupadas en tres conglomerados. El primero concerniente a la globalización e internacionalización de las universidades (ejemplo: Jöns y Hoyler, 2013); el segundo relacionado a la evaluación del rendimiento de las instituciones de educación superior (por ejemplo: Billaut et al., 2010), y el tercero referido a la calidad y las políticas de educación superior (ejemplo: Cantwell y Taylor, 2013). Finalmente, el tercero clúster, de color azul, contiene 10 palabras que se reagrupan en dos conglomerados. El primero se focaliza en la información que entregan los rankings (ejemplo: Dill y Soo, 2005) y el segundo en la evaluación a los rankings universitarios (ejemplo: Pusser y Marginson, 2013).

\section{DISCUSIÓN FINAL}

A través de técnicas bibliométricas, se analizaron las características de la producción científica sobre RU publicada en la WoS durante los años 1988 y 2018. Se pudo constatar que a partir del año 2004, luego de la aparición del primer ranking universitario global, los estudios sobre RU aumentaron significativamente, reflejando el interés que ha generado el tema en la comunidad científica. En cuanto a las zonas geográficas, se determinó que las investigaciones se han realizado principalmente en organizaciones y países europeos, asiáticos y de Norte América. De igual forma, se constató que un número importante de artículos estudian casos específicos de países de estas mismas regiones. Por otra parte, en América Latina, región con baja presencia en los rankings globales (King-Domínguez et al., 2018), se ha desarrollado un número escaso de investigaciones sobre el tema.

En lo que se refiere a las revistas, las principales áreas sobre las que publican son "educación e investigación en educación", "ciencias de la computación" y "ciencias de la informática y ciencias de la biblioteca". Esto delata las disciplinas sobre las cuales se ha investigado en relación a los RU. Por otra parte, se detectaron aquellas que son centrales en el campo de la investigación estudiado, ya que 13 revistas concentra un tercio 
de los artículos referidos al tema estudiado, muchos de los cuales pueden considerarse de alto impacto según el número de citas que tienen. El origen de este grupo de revistas es principalmente europeo, al igual que muchas de las organizaciones que han trabajado en la materia. Esta situación muestra el alto interés que suscita la temática de los RU en este continente.

En relación a las líneas de investigación, se pueden distinguir tres grupos, cada uno dividido en subgrupos. El primer grupo se investiga aspectos propios de la investigación (medición de la productividad de la investigación; impacto de la investigación; divulgación del conocimiento). El segundo se enfoca en la educación superior y sus instituciones (globalización e internacionalización de las universidades; evaluación del rendimiento de las instituciones de educación superior; y calidad y las políticas de educación superior. Por último, el tercer clúster analiza los distintos tipos de RU (información entregada por los rankings y la evaluación de los RU).

\section{CONCLUSIONES}

De acuerdo al trabajo presentado y a los resultados obtenidos, se pueden plantear las siguientes conclusiones principales:

1. El análisis bibliométrico permitió definir la evolución que ha tenido la investigación sobre RU. A pesar de que se han publicado artículos por más de 40 años, el mayor interés en el área surge a partir del año 2004, luego de la publicación del ranking ARWU, primero a nivel global.

2. La mayor productividad científica en RU se concentra en Europa, seguida de Asia y América. Coincidentemente, los autores con mayor número de publicaciones están adscritos a instituciones europeas. Entre los más citados destacan Antony F.J. Van Raan y David D. Dill, cuyos trabajos pueden ser considerados seminales en el tema, según el número de citas recibidas. El análisis de co- palabras evidenció tres grandes líneas de investigación, siendo la más importante la relacionada a la medición de la productividad de la investigación.

3. La presente estudio entrega la evolución de esta línea de investigación a través del tiempo, la que puede utilizarse como guía para nuevos investigadores o para quienes deseen profundizar en el tema y requieran conocer sobre los artículos, autores, países, revistas e instituciones más influyentes.

4. El estudio se limita a utilizar la base de datos WoS, excluyendo otras bases internacionales.

\section{REFERENCIAS}

Abramo G. y D'Angelo, C.A., How do you define and measure research productivity? Scientometrics, 101(2), 1129-1144 (2014)

Aguilló I., Bar-llan, J., Levene, M. y Ortega, J., Comparing university rankings, Scientometrics, 85(1), 243-256 (2010)

Barra, A.M., La importancia de la productividad científica en la Acreditación Institucional de Universidades Chilenas, Formación Universitaria, 12(3), 101-110 (2019)

Billaut, J.C., Bouyssou, D. y Vincke, P., Should you believe in the Shanghai ranking?, Scientometrics, 84(1), 237-263 (2010)

Buela-Casal, G., Gutiérrez-Martínez, O., Bermudez-Sánchez, M. y Vadillo-Muñoz, O., Comparative study of international academic rankings, Scientometrics, 71(3), 349-365 (2007)

Cantwell, B. y Taylor, B.J., Global Status, Intra-Institutional Stratification and Organizational Segmentation: A TimeDynamic Tobit Analysis of ARWU Position Among U.S. Universities, Minerva, 51(2), 195-223 (2013)

Dill, D.D. y Soo, M., Academic quality; league tables, and public policy: A cross-national analysis of university ranking systems, Higher Education, 49 (4), 495-533 (2005)

Espeland, W.N. y Suader, M., Rankings and reactivity: how public measures recreate social worlds, American Journal of Sociology, 113(1), 1-40 (2007)

Hazelkorn, E., Learning to live with League Tables and Ranking: The Experience of Institutional Leaders. Higher Education Policy, 21(2), 193-216, (2008)

Hazelkorn, E., Rankings and the reshaping of higher education: the battle for world class excellence. In Springer (Ed.), Studies in Higher Education, Macmillan, Palgrage, (2011)

Herrera, J., Larrán, M., Lechuga, M. y Martínez-Martínez, D., Evolución de la literatura sobre la responsabilidad social en pymes como disciplina científica, Revista Europea de Dirección y Economía de la Empresa, 24(2), 117-128 (2015)

Hirsch, J.E., An index to quantify an individual's scientific research output, Proceedings of the National Academy of Sciences, 102(46), 16569-16572 (2005) 
Huang, C.Y. y Ho, Y.S., Historical research on corporate governance: A bibliometric analysis, African Journal of Business Management, 5(2), 276-284 (2011)

Jöns, H. y Hoyler, M. ,Global geographies of higher education : the perspective of world university rankings. Geoforum, 46 , 45-59 (2013)

King-Domínguez, A, Llinàs-Audet, X. y Améstica-Rivas, L., Rankings universitarios como medida de calidad: análisis comparado en Latinoamérica, Revista Venezolana de Gerencia, 23(1), 218-237 (2018)

Liu, N.C. y Cheng, Y., The Academic Ranking of World Universities, Higher education in Europe, 30(2), 127-136 (2005)

Marginson, S. y Van der Wende, M., To Rank or To Be Ranked: The Impact of | Rankings in Higher Education, Journal of Studies in International Education, 11(3-4), 306-329 (2007)

O'Loughlin, D., MacPhail, A. y Msetfi, R., Studies in Higher Education The rhetoric and reality of research reputation : "fur coat and no knickers", Studies in Higher Education, 40(5), 806-820 (2015)

Ortiz-de Urbina-Criado, M., Nájera-Sánchez, J.J. y Mora-Valentín, E.M., A Research Agenda on Open Innovation and Entrepreneurship : A Co-Word Analysis, Administrative Sciences, 8(3) 34, 1-17 (2018)

Pusser, B. y Marginson, S., University Rankings in Critical Perspective, The Journal of Higher Education, 84(4), 544-568 (2013)

Rauhvargers, A., Global university rankings and their impact. Leadership for WorldClass Universities Challenges for Developing Countries, European University Association (2011)

Saisana, M., d'Hombres, B. y Saltelli, A., Rickety numbers: Volatility of university rankings and policy implications, Research Policy, 40(1), 165-177 (2011)

Salmi, J., El desafío de crear universidades de rango mundial, World Bank, Mayol Ediciones (2009)

Salmi, J. y Saroyan, A., League Tables as Policy Instruments, Higher Education Management and Policy, 19(2), 1-38 (2007)

Thelwall, M. y Kousha, K., ResearchGate: Disseminating, Communicating, and Measuring Scholarship? Mike, International Review of Research in Open and Distance Learning, 14(4), 90-103 (2008)

Torres-Salinas, D., Moreno-Torres, J., Delgado-López-Cózar, E. y Herrera, F., A methodology for Institution-Field ranking based on a bidimensional analysis: The IFQ2A index, Scientometrics, 88(3), 771- 786 (2011)

Tsay, M. y Li, C., Bibliometric analysis of the journal literature on women's studies, Scientometrics, 113(2), 705-734 (2017) Van Eck, N.J. y Waltman, L., VOSviewer Manual. Leiden (2017)

Van Raan, A. F., Fatal attraction: Conceptual and methodological problems in the ranking of universities by bibliometric methods, Scientometrics, 62(1), 133-143 (2005)

Waltman, L., Calero-Medina, C. y otros ocho autores, The Leiden Ranking 2011 / 2012 : Data collection, indicators, and interpretation, Journal of the American Society for Information Science and Technology, 63(12), 2419-2432 (2012) 